\title{
The Impact of Construction Industry Structure Optimization on the Economy of Metropolitan Area - Taking Hefei and Nanjing Metropolitan Area as Examples
}

\author{
Cui Juyan ${ }^{* 1}$, Wang Ziyang ${ }^{1}$, Chen feiyang ${ }^{1}$ \\ ${ }^{1}$ School of Architecture and Engineering, Tongling College, 244061Tongling, Anhui , China
}

\begin{abstract}
This research selected Hefei and Nanjing metropolitan area as the research object to analyze the optimization of construction industry structure in Hefei and Nanjing metropolitan area in 2018. Firstly, the construction industry structure rationalization analysis, heightened analysis, specialization analysis and similarity analysis were conducted for Hefei and Nanjing metropolitan areas, then the theory of construction industry structure optimization analysis was derived for Hefei and Nanjing metropolitan areas, and then ArcGIS technology was used to draw the spatial distribution map of construction industry structure in the two metropolitan areas to provide theoretical support for industrial structure optimization and high-quality construction of Hefei metropolitan area. Finally, the analysis concluded that in the process of synergistic development, the Hefei metropolitan area should focus on strengthening the synergistic development with Nanjing metropolitan area, so that the Yangtze River Delta region can achieve the optimization of economic structure preferably, advanced industrial base and modernization of industrial chain, and enhance the coordination of urban and rural regional development, thus making significant progress in the construction of modernized economic system.
\end{abstract}

\section{Introduction}

The concept of metropolitan area was proposed by the National Development and Reform Commission in 2019 in the Guidance on Fostering the Development of Modernized Metropolitan Areas. Metropolitan area is a special form of territorial spatial organization, which is the result of the joint action of economy, politics, culture and society. Among them, the construction industry, as a pillar industry of China's national economy, is an essential part of the comprehensive construction of a modernized socialist country[1][2]. Up to now, China has not established the planning and target on promoting the modernization of construction industry, and a set of policy system needed to promote the rationalization, heightening and modernization of construction industry structure which is yet to be improved, while a systematic construction industry system is missing as a strong support. This situation shows that Chinese construction industry is still largely not free from the dependence on the traditional path, on the other hand, it also shows that the development of the construction industry has entered an important historical juncture that must be transformed and upgraded[3]. This requires us to re-examine the traditional "industry" way of thinking and the shortcomings of the development path, from the perspective of "industrial structure optimization", indepth study of Chinese construction industry, a series of fundamental understanding and internal law issues, which it is of great practical significance to explore the way of sublimation of the modernization of Chinese construction industry.

After the Nanjing metropolitan area plan was announced and approved by the National Development and Reform Commission in 2021, it will provide a demonstration and accumulate experience for the construction of new metropolitan areas in China, and also should require Jiangsu and Anhui provinces to build the Nanjing metropolitan area into a modern metropolitan area with national influence. With the development of national economy, the market demand of construction industry is also increasing. I. Stumpf of the University of London defined the criteria for different types of construction enterprises in the British construction industry. In the construction industry, organizations operate in a dynamic, competitive and unpredictable environment. Morcos Maqed S.[4] pointed out that the reliability of organizational structures in the construction industry can be assessed and managed through a decision support system that uses a combination of qualitative and quantitative methods using component parametric analysis and fault tree analysis. In 2000, I. Stumpf [5] analyzed the size structure of enterprises in the UK construction industry from 1981 to 1996 and found that the relative number and market share of medium-sized construction enterprises in the market had decreased, and that the lack of the ability of medium-sized construction enterprises to develop international markets and the

*Corresponding author: icy-cui@vip.126.com 
ability of small enterprises to adapt to the market flexibly and quickly was also an important reason. In the United States, D. Arditi [6] analyzed the failure and reasons for the exit of construction firms in the United States from 1989 to 1993 . There is considerable domestic research on the industrial structure of the construction industry. Three scholars, including Chianq Y. H. of the Hong Kong Polytechnic University[7], studied the market structure of the construction industry in Hong Kong and measured the Herfindahl indices of the civil engineering contracting market, the public housing market, and the private housing contracting market in Hong Kong, and judged from the size of the indices that the highest degree of concentration was in the civil engineering contracting market, followed by the public housing market, and the lowest was in the private housing contracting market. Xibao[8] conducted an international comparative study mainly on macro issues of the construction industry, and conducted an in-depth study on the production methods of the construction industry, the market supply and demand of the construction industry, the growth fluctuations of the construction industry, the development of the industrial structure and the coordinated development of the construction industry and the national economy. Jin Weixing et al.[9], after analyzing the current situation of industrial organization in China's construction industry, argued that improving the competitiveness of China's construction industry should start from the three aspects. Chen Y[10] studied the ways of adjusting the industrial structure of China's construction industry and proposed some method. Based on the research of many scholars on the industrial structure of construction industry, this study will start from the real situation of the construction market structure of Hefei and Nanjing, using methods such as industrial structure optimization theory, analyzing the integrated development of Nanjing metropolitan area, optimizing the structure of construction industry in Hefei, and use ArcGIS to draw the industrial structure distribution map, studying the core of the construction market structure and the basic form of optimizing the market structure.

\section{Overview of the study area and data sources}

\subsection{Site description}

Hefei metropolitan area, including Hefei, Huainan, Liuan, Chuzhou, Wuhu, Maanshan, Bengbu, Tongcheng (county-level city) seven prefecture-level cities, located in the middle and lower reaches of the Yangtze River cities, located in the middle and lower reaches of the Yangtze River along the western end of the Yangtze River Delta, is one of the five major metropolitan areas of the Yangtze River Delta city group, the Yangtze River Delta to the central and western extension of an important hub and the western gate, the regional area accounted for $40.6 \%$ of the province, the population accounted for $43.2 \%$ of the province. Until 2019, the Hefei metropolitan area achieved a regional GDP of
2,340.2 billion yuan and a fiscal revenue of 344.07 billion yuan. And Nanjing metropolitan area is a core area formed in the middle and lower reaches of Yangtze River and combined with coastal cities, straddling Jiangsu and Anhui provinces, including Nanjing, Yangzhou, Zhenjiang and Huaian in Jiangsu Province and Maanshan, Chuzhou, Wuhu and Xuancheng in Anhui Province. Under the leading role of regional development strategy, Nanjing metropolitan area geographically connects four cities in Jiangsu Province belonging to Yangtze River Delta region and four cities in Anhui Province belonging to the central region[11].

\subsection{Data sources}

The basic data of economic development of Hefei and Nanjing metropolitan area are obtained from the statistical yearbooks of each city in Hefei and Nanjing metropolitan area, and the basic data include GDP per capita, contribution rate of three industries, personnel composition of three industries, construction output value, and personnel composition of construction industry in each city. By analyzing the economic development data and construction industry-related data of Hefei and Nanjing metropolitan areas in 2018, the industrial structure of the construction industry in Hefei and Nanjing metropolitan areas is studied by using the rationalization discrimination, heightened discrimination and specialization discrimination of industrial structure optimization.

\section{Research methods and data}

\subsection{Criteria for discriminating industrial structure optimization}

\subsubsection{Rationalized discrimination}

The American economist Simon Kuznets put forward the standardized structure in 1971. In the process of determining this structure, if the industrial structure of a country or a region differs from the "standard structure", it means that the industrial structure of the region or the country deviates from the development trajectory of most countries, and to a certain extent The industrial structure is unreasonable, and vice versa[3]. In this paper, we refer to the internationally recognized criteria of industrial structure of countries in different development stages summarized by the World Bank in 1997.

\subsubsection{Heightened discrimination}

Firstly, analysis of the softening degree of industrial structure. The softening rate is generally used to measure the softening degree[3] of the industrial structure, and there are two general formulas for calculating the softening rate, and this paper uses two indicators to analyze for the convenience of finding data, namely, the ratio of the output value and employment of the tertiary industry to GDP and total employment respectively, the 
ratio is $a, b$, then c can be used to express the softening rate that is a and the average of $b$.

Secondly, Industrial structure analysis. By measuring the level of industrial structure, the level of industrial structure heightening can be better reflected. The prerequisite for the measurement of industrial structure is the need to construct the industrial structure level coefficient, which is calculated by the formula

$$
H=\sum_{\mathrm{i}=1}^{\mathrm{n}} \mathrm{k}_{\mathrm{i}} \sqrt{p_{i} / l_{i}}
$$

Where $\mathrm{n}$ denotes the number of industrial sectors in a region, $k_{i}$ denotes the proportion of output value of industry $i, p_{i}$ and $l_{i}$ represent the output value of industry $i$ and the number of people employed in industry i, respectively, and $\mathrm{p}_{\mathrm{i}} / \mathrm{l}_{\mathrm{i}}$ denotes the labor productivity of industry $i$. The root sign is the industry level coefficient of industry $i$, denoted by $A_{i}$, and the multiplication of $k_{i}$ and $\mathrm{Ai}$ is the contribution of an industry in the industrial structure level, denoted by $\mathrm{B}_{\mathrm{i}}$.

\subsubsection{Specialized discriminations}

Industrial specialization is mainly measured by the "location entropy"[12] index, which can be greater than 1 or less than 1 . If it is greater than 1 , it means that the industry has a high degree of specialization, and vice versa, it has a low degree of specialization. The formula is

$$
\mathrm{R}=\left(A_{i} / A_{i}\right) /\left(B_{j} / B\right)
$$

Where $A_{i j}$ is the output value of industry $j$ in region $i$ and $A_{i}$ is the gross product of region $i$, then $A_{i j} / A_{i}$ is the contribution rate of industry $\mathrm{j}$ in region $\mathrm{i}$ : coincidentally, the output value of industry $\mathrm{j}$ in the country and $\mathrm{B}$ is the national gross product, then $B_{j} / B$ is the contribution rate of industry $\mathrm{j}$ in the country.

\subsection{Using ArcGIS to map the spatial distribution of industrial structures within the two metropolitan areas}

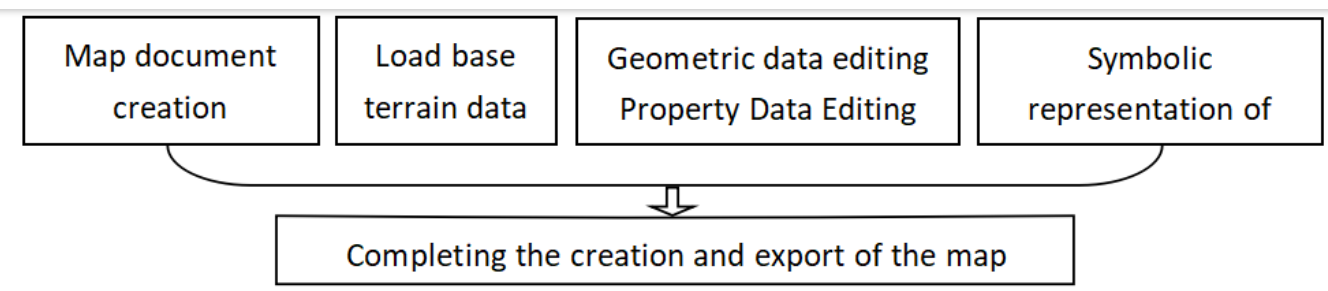

Figure 1. Steps to create a thematic map using ArcGIS

\section{Analysis of results}

\subsection{Analysis of industrial structure rationalization}

Combining the criteria for determining the "standard structure" and Table 1, the following two conclusions can be drawn.

First, in terms of economic development level, only six cities in the Hefei-Nanjing metropolitan area, namely Hefei, Wuhu, Maanshan, Nanjing, Zhenjiang and Yangzhou, have per capita GDP exceeding the national per capita GDP level, which shows that there is a certain imbalance in the current economic development level of Hefei and Nanjing metropolitan area.

Second, the industrial and employment structures of Hefei and Nanjing metropolitan areas show inconsistencies. The composition of the output value of primary industry and the proportion of employed persons in Hefei and Nanjing metropolitan areas are significantly lower, while the tertiary industry is significantly higher and the secondary industry is second, showing their underdevelopment, which is consistent with their overall industrial structure development trend, i.e. the development trend of "three, two, one".

Table1. Summary of GDP per capita and three industries in Hefei and Nanjing metropolitan areas, 2018

\begin{tabular}{|l|l|l|l|l|l|l|l|}
\hline City Indicators & $\begin{array}{l}\text { GDP per } \\
\text { capita (yuan) }\end{array}$ & \multicolumn{2}{l}{$\begin{array}{l}\text { Composition of the three industries (\%) } \\
\text { Trimary industry Secondary industry }\end{array}$} & \multicolumn{2}{l|}{$\begin{array}{l}\text { Composition of employees (\%) } \\
\text { Primary industry Secondary industry } \\
\text { Tertiary industry }\end{array}$} \\
\hline Hefei & 114680.0 & 3.20 & 36.90 & 56.90 & 13.00 & 34.90 & 52.10 \\
\hline Xuancheng City & 50065.0 & 10.3 & 48.7 & 41.00 & 34.16 & 28.12 & 40.42 \\
\hline Huainan City & 37140.0 & 10.00 & 40.70 & 49.30 & 28.40 & 28.70 & 43.00 \\
\hline Lu'an City & 26731.0 & 15.3 & 32.9 & 44.1 & 35.2 & 21.6 & 43.1 \\
\hline Chuzhou City & 43999.0 & 14.70 & 55.00 & 30.30 & 33.80 & 31.20 & 35.00 \\
\hline Wuhu City & 88085.0 & 4.00 & 52.20 & 43.80 & 22.20 & 28.70 & 49.10 \\
\hline Ma'anshan City & 82938.0 & 23.30 & 29.50 & 47.20 & 23.30 & 25.90 & 47.20 \\
\hline Bengbu & 50662.0 & 12.10 & 44.50 & 43.40 & 42.60 & 21.90 & 35.50 \\
\hline Nanjing & 152886.0 & 2.10 & 35.90 & 62.00 & 5.50 & 35.60 & 58.90 \\
\hline Zhenjiang & 126906.0 & 3.70 & 48.40 & 47.90 & 11.22 & 43.69 & 45.09 \\
\hline Yangzhou City & 142020.0 & 5.00 & 47.99 & 47.10 & 13.92 & 42.94 & 43.13 \\
\hline Huai'an & 73204.0 & 10.00 & 41.80 & 48.20 & 6.80 & 51.50 & 41.70 \\
\hline
\end{tabular}




\subsection{Analysis of industrial structure heightening}

\subsubsection{Analysis of the degree of softening of industrial structure}

In this paper, based on the relevant statistical yearbooks, the ratios of the output value and the number of employed persons in the construction industry to GDP and the total number of employed persons are calculated, and the ratios are $a$ and $b$ respectively, while the softening rate is the average of these two ratios, which is expressed by c. The values are summarized as shown in Table 2, from which the following two conclusions can be drawn.

First, the industrial structure softening rate of the Hefei-Nanjing metropolitan area in 2018 is slightly better than that of the whole country, and according to the discriminatory criteria, the construction industry in the eight cities of Hefei, Huainan, Wuhu, Maanshan, Nanjing, Huaian, Yangzhou and Zhenjiang in the Hefei-Nanjing metropolitan area is a low softening industry. The construction industry in the remaining four cities belongs to the hard industry, and the contribution rate of their construction industry to economic development and the proportion of employment in this industry are mostly below $50 \%$, which shows that the economic development of Hefei and Nanjing metropolitan area does not rely mainly on the construction industry to pull.

Second, from the city's perspective, Hefei's construction industry belongs to the ranks of low-soft industries, but is fast approaching the ranks of high-soft industries, with its construction industry contribution rate and employment ratio exceeding half, indicating that the current construction industry in Hefei has a significant pulling effect on economic development.

Table2. Summary of the softening coefficient of the construction industry structure in Hefei and Nanjing metropolitan area

\begin{tabular}{|c|c|c|c|}
\hline City Indicators & $\mathrm{a}$ & $\mathrm{b}$ & $\mathrm{c}$ \\
\hline Hefei & 59.94 & 52.10 & 56.02 \\
\hline Xuancheng City & 43.12 & 40.42 & 41.77 \\
\hline Huainan City & 46.57 & 41.50 & 44.04 \\
\hline Lu'an City & 20.83 & 43.1 & 31.97 \\
\hline Chuzhou City & 36.12 & 35.00 & 35.56 \\
\hline Wuhu City & 43.80 & 49.10 & 46.45 \\
\hline Ma'anshan City & 41.90 & 47.20 & 44.55 \\
\hline Bengbu & 43.40 & 35.50 & 39.45 \\
\hline Nanjing & 44.12 & 61.04 & 52.58 \\
\hline Zhenjiang & 47.78 & 46.02 & 46.90 \\
\hline Yangzhou City & 47.01 & 40.17 & 43.59 \\
\hline Huai'an & 48.16 & 41.49 & 44.83 \\
\hline
\end{tabular}

\subsubsection{Industrial structure level analysis}

Based on the relevant statistical yearbooks, the industrial structure level coefficient $\mathrm{H}$ is calculated (as shown in Table 3). From the table, we can see that: the industrial structure level of Hefei metropolitan area is much higher than that of Nanjing metropolitan area, and its $\mathrm{H}$ value is the highest at 253.08; the highest in Nanjing metropolitan area is 114.58, while Huai'an is the lowest, but the industrial structure level of all cities shows an upward trend compared with previous years, and its industrial structure heightened level is increasing.

Table3. Industrial structure level of construction industry in Hefei and Nanjing metropolitan area

\begin{tabular}{|c|c|c|c|}
\hline Region & Industry output value share (\%) & Industry level coefficient & Industry Structure Level \\
\hline Hefei & 15.52 & 699.76 & 108.60 \\
\hline Xuancheng City & 7.33 & 418.24 & 30.66 \\
\hline Huainan City & 7.28 & 563.07 & 40.99 \\
\hline Lu'an City & 7.70 & 511.85 & 39.41 \\
\hline Chuzhou City & 23.93 & 604.16 & 39.78 \\
\hline Wuhu City & 5.80 & 685.82 & 48.50 \\
\hline Ma'anshan City & 6.10 & 795.05 & 253.08 \\
\hline Bengbu & 36.59 & 691.67 & 19.88 \\
\hline Nanjing & 5.20 & 382.30 & 92.61 \\
\hline Zhenjiang & 13.61 & 680.45 & 16.73 \\
\hline Yangzhou City & 6.23 & 268.57 & 15.02 \\
\hline Huai'an & 6.70 & 224.15 & \\
\hline
\end{tabular}




\subsection{Analysis of industrial structure specialization}

The following is an analysis of the construction industry in the Hefei and Nanjing metropolitan areas. In general, the degree of specialization of the construction industry in Hefei and Nanjing metropolitan area are relatively low, but the degree of specialization of the construction industry in Bengbu and Chuzhou cities in the two metropolitan areas is higher. There are three construction industries with location quotient greater than 0.5 , namely Hefei, Bengbu and Chuzhou, and the degree of specialization of the construction industry in the remaining nine cities is less than 0.5 , but shows an upward trend compared with previous years.

Second, from the two provinces, the highest degree of structural specialization of the construction industry in
Bengbu, Anhui Province is 1.45 , followed by Chuzhou. It can be seen that the current development of Bengbu and Chuzhou city focus on the construction industry, within Jiangsu Province, the highest degree of structural specialization of the construction industry in Zhenjiang city is 0.54 , the remaining three cities have a low degree of structural specialization of the construction industry, between $0.2-0.3$, it can be seen that the current development of Zhenjiang city focus is favoring the construction industry

\subsection{Using ArcGIS to map the spatial distribution of the construction industry structure in two major metropolitan areas}

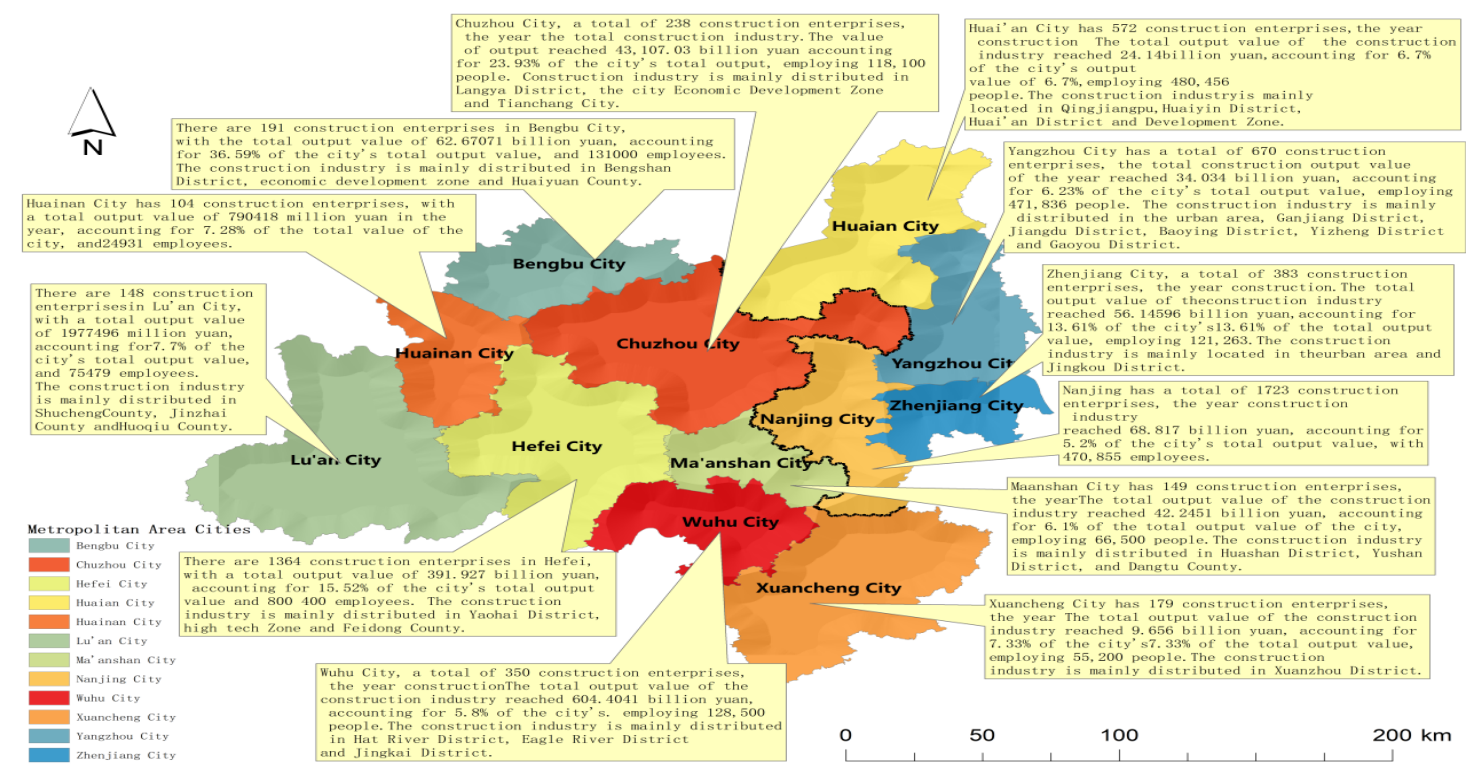

Figure 2. Industrial structure distribution of construction industry in Hefei and Nanjing metropolitan area

The following is an analysis of the spatial distribution of the industrial structure of the construction industry in the metropolitan area of Hefei and Nanjing.

First of all, the overall number of construction enterprises, total construction output value, construction employees or the total value of construction output value in the Hefei metropolitan area is higher than that in the Nanjing metropolitan area, and the construction industry in Hefei within the Hefei metropolitan area is in the leading position in the province and is the leader of the construction market in Anhui Province. In 2018, Hefei contributed about fifty percent of the construction industry with the number of units accounting for less than forty percent of the province's total output value, enterprise assets, business income, and employees, etc. Among the top 50 enterprises in the province, Hefei occupied 39, accounting for more than three quarters of the province. While Nanjing City in Nanjing metropolitan area is not the leader of the construction market in its metropolitan area, as of the end of 2018, the number of construction enterprises in Yangzhou City is 670, although not as many as the number of construction enterprises in Nanjing City, but the number of construction employees in Yangzhou City at the end of the year is 10,400,000 more than Nanjing City, a total of 896,300 people engaged in the construction industry, Yangzhou City's total construction output value in 2018 than Nanjing City 8.127 billion yuan more. The first three data may not show that Yangzhou's construction industry is stronger than Nanjing's, but it can be judged based on the completed area of housing construction. 106,498,600 square meters of housing construction was completed in Yangzhou at the end of 2018, while Nanjing only had $58,484,600$ square meters, a difference of nearly one times between the two cities. The comprehensive first four indicators show that the construction industry in Nanjing is less developed than that in Yangzhou. The construction industry is the pillar industry, people-rich industry and advantageous industry of Yangzhou, as the provincial government named the "first batch of strong construction city", Yangzhou municipal party committee and municipal government attached great importance to the development of the construction industry, as early as 2012, the fourth plenary session of the sixth session of the Yangzhou Municipal Party Committee clearly positioned the construction industry as Yangzhou is focusing on one 
of the four basic industries[14]. In recent years, Yangzhou construction industry takes the scientific outlook on development as the leader, seizing the opportunity to deepen reform, speeding up structural adjustment, focusing on cultivating the market, and strengthening brand building. The scale of the construction industry has a new level every year, and the strength of construction enterprises is constantly enhanced, and their contribution to the construction industry is also increasing.

\section{Conclusion}

(1) The industrial structure and employment structure of Hefei and Nanjing metropolitan area are not coordinated. Half of the cities in the Hefei metropolitan area show that the output value composition and employment proportion of primary and tertiary industries are obviously low, while secondary industries are obviously high, which is consistent with their "two, three, one"development model[13]. This is in line with its "two, three, one" development model, which is gradually developing in the direction of "three, two, one" according to its development stage. The development pattern of the other half of the cities is already "three, two, one" in line with the general law of industrial structure. The development trend of industrial structure in Nanjing metropolitan area is similar to that of Hefei metropolitan area, and according to its development pattern, it has started to evolve in the direction of "three, two, one", which is consistent with the general rule of industrial structure evolution.

(2) The industrial structure of the Hefei and Nanjing metropolitan areas is increasing in heightened level. In terms of the construction industry in the two metropolitan areas, only the construction industry in eight cities, namely Hefei, Huainan, Wuhu, Maanshan, Nanjing, Huai'an, Yangzhou and Zhenjiang, is a low-soft industry, but according to its contribution to the economic development of this metropolitan area and its share of employment, it is clear that the construction industry has a significant contribution to the innovative Yangtze River Delta only Hefei plays a significant role in the economic development of the metropolitan area, and the contribution of the construction industry to the economic development and the share of employment in this industry are mostly higher than $50 \%$. The construction industry in the remaining seven cities does not play a critical role in the economic development, and its development needs to be further strengthened.

(3) Comparing the degree of specialization of Hefei and Nanjing metropolitan areas, it can be seen that the degree of specialization of the construction industry in Hefei metropolitan area is higher than that in Nanjing metropolitan area. This shows that the development of Hefei metropolitan area is currently focused on the construction industry, while the development of Nanjing metropolitan area is changing its focus to the construction industry.

(4) According to the theory of industrial structure optimization, it is known that the rationalization and heightening of industrial structure have different effects on the optimization of industrial structure in Hefei and
Nanjing metropolitan areas. Improving the level of industrial structure rationalization and heightening can promote the development of industrial structure of the two metropolitan areas to a good posture on the whole.

\section{Policies and Recommendations}

(1) Optimize enterprise qualification work. Assist highgrade qualified enterprises to become bigger and stronger, support large enterprises to carry out horizontal acquisitions and mergers, vertical chain integration, strategic reorganization of equity, and guide leading enterprises to extend the whole industry chain from traditional construction general contracting to investment design, development and construction, operation and maintenance, parts production, etc. Encourage small and medium-sized enterprises to take the road of specialization and refinement, targeting niche areas, and select a number of small and medium-sized micro construction enterprises with outstanding performance and good growth in a planned and focused manner.

(2) Encourage scientific and technological innovation in construction. Increase support for scientific research investment in the construction industry, and actively promote the construction of independent innovation capabilities of enterprises, encourage construction enterprises to declare new technology enterprises, the establishment of technology centers, support the recommended enterprises to declare major science and technology special projects and the transformation of scientific and technological achievements to promote the project. Increase the training of BIM technology talents, and comprehensively enhance the application of BIM technology in the field of engineering construction.

(3) Increase policy support. Support the development of the construction industry in Hefei City, Bengbu City and Yangzhou City, on the basis of this triangular structure can drive the development of the construction industry in other cities in the metropolitan area. Encourage foreign construction industry leaders to settle in Hefei and Nanjing metropolitan area, meet the relevant conditions required to give a one-time government financial incentives and credit incentives. Encourage local construction enterprises to increase production and generate income, and give one-time government financial incentives, to enterprises that have won the "Luban Award", "National Award of Excellence" and "Zhan Tianyou Award".

(4) Improve the construction work mechanism. Strengthen the city (county) district, the development zone of the construction industry development indicators assessment efforts, the formation of the work of the first place atmosphere and rewards and punishments are clear assessment guide. Establish a joint meeting system of the member units to deepen the construction industry "management and service" reform, and promote the relevant government departments at all levels, between the government and enterprises, the cooperation mechanism between banks and enterprises to improve service effectiveness. 


\section{Funding:}

The research was supported by the Anhui Provincial Philosophy and Social Science Planning Youth Project (Research on Spatial Integration of Hefei Metropolitan Area and Nanjing Metropolitan Area) (AHSKQ2020D50)

\section{References}

1. Chen Hongyan. Analysis of the current situation of Jiangxi construction industry development and adjustment countermeasures [J]. Jiangxi Social Science, 000,006:167-170,(2006).(In Chinese)

2. Zhang Haoran, Yi Baozhong. The Effect of Industrial Structure Variation on Urban Employment in China: A Spatial Panel-Data Study[J]. Industrial Economics Research, 000,003:50-55,(2011).(In Chinese)

3. Liao Yuping. Research on industrial restructuring strategy of construction industry $[\mathrm{J}]$. Construction Economy,03:5-9,(2005). (In Chinese)

4. Morcos Maqed S, Sinqh Gurdev. Decision support system or reliability assessment of management structures of organizations in the construction industry $[\mathrm{J}]$, Congress on Computing in Civil Engineering, Proceedings, v 2, p 1577-1583,(1995).

5. I Stumpf. Competitive Pressures on Middle-market Contractors in the UK[J]. Engineering Construction and Architectural Management,7,2: 159-168,(2000).

6. D Arditi. A Koksal and S Kale. Business Failures in the Construction Industry[J]. Engineering Construction and Architectural Management ,7,2: 120-132, (2000).

7. Y H Chiang. B S Tang and w Y Leung. Market Structure of the Construction Industry in Hong

Kong[J]. Construction management and Economics., 19,5:675-687,(2001).

8. Xibao. Comparative study of construction industry [d]. Doctoral dissertation of Harbin University of architecture, (1999)(In Chinese)

9. Jin Weixing, et al. On the restructuring of China's construction industry and its enterprises [J], construction economy, 4: 8-14,(1999).(In Chinese)

10. Chen Yancun. Current situation, problems and adjustment approaches of China's construction industry structure [D], master's thesis of Xiamen University, (2001)(In Chinese)

11. Yang Qian,Tang Xianglong. Research on the comparative advantages of urban industries in Hexi Corridor based on location entropy theory[J]. Industrial Development, No. 4,90-95(2019). (In Chinese)

12. Jin Jiawen. Research on industrial collaboration relationship in Hefei metropolitan area from the perspective of central cities [D]. Anhui Agricultural University, (2018).(In Chinese)

13. Sui Zhi-kuan. A comparative study on the relationship and industries between major and minor cities in Beijing-Tianjin-Hebei and Yangtze River
Delta metropolitan areas[D]. Central University of Finance and Economics, (2015).(In Chinese) 